\title{
A quantitative trait locus on Bos taurus autosome 17 explains a large proportion of the genetic variation in de novo synthesized milk fatty acids
}

\author{
S. I. Duchemin,${ }^{*} \dagger^{1}$ M. H. P. W. Visker, ${ }^{*}$ J. A. M. Van Arendonk, ${ }^{*}$ and H. Bovenhuis ${ }^{*}$ \\ *Animal Breeding and Genomics Centre, Wageningen University, PO Box 338, 6700 AH Wageningen, the Netherlands \\ †Department of Animal Breeding and Genetics, Swedish University of Agricultural Sciences, Uppsala, Sweden
}

\begin{abstract}
A genomic region associated with milk fatty acid (FA) composition has been detected on Bos taurus autosome (BTA)17 based on 50,000 (50K) single nucleotide polymorphism (SNP) genotypes. The aim of our study was to fine-map BTA17 with imputed 777,000 (777K) SNP genotypes to identify candidate genes associated with milk FA composition. Phenotypes consisted of gas chromatography measurements of $14 \mathrm{FA}$ based on winter and summer milk samples. Phenotypes and genotypes were available on 1,640 animals in winter milk, and on 1,581 animals in summer milk samples. Single-SNP analyses showed that several SNP in a region located between 29.0 and $34.0 \mathrm{Mbp}$ were in strong association with C6:0, C8:0, and C10:0. This region was further characterized based on haplotypes. In summer milk samples, for example, these haplotypes explained almost $10 \%$ of the genetic variance in $\mathrm{C} 6: 0,9 \%$ in $\mathrm{C} 8: 0$, $3.5 \%$ in $\mathrm{C} 10: 0,1.8 \%$ in $\mathrm{C} 12: 0$, and $0.9 \%$ in $\mathrm{C} 14: 0$. Two groups of haplotypes with distinct predicted effects could be defined, suggesting the presence of one causal variant. Predicted haplotype effects tended to increase from C6:0 to C14:0; however, the proportion of genetic variance explained by the haplotypes tended to decrease from C6:0 to C14:0. This is an indication that the quantitative trait locus (QTL) region is involved either in the elongation process or in early termination of de novo synthesized FA. Although many genes are present in this QTL region, most of these genes on BTA17 have not been characterized yet. The strongest association was found close to the progesterone receptor membrane component 2 (PGRMC2) gene, which has not yet been associated with milk FA composition. Therefore, no clear candidate gene associated with milk FA composition could be identified for this QTL.
\end{abstract}

Key words: milk fatty acid composition, dairy cattle, candidate genes, high-density genotyping

Received March 28, 2014

Accepted July 23, 2014.

${ }^{1}$ Corresponding author: sandrine.duchemin@wur.nl

\section{INTRODUCTION}

Bovine milk fat is composed of more than 400 different FA, many of which are still unidentified (Jensen, 2002). Fatty acids may differ in the number of carbons and this difference can be related to the origin of the FA. Most short-chain FA have fewer than 12 carbons that are mainly elongated from acetate by de novo synthesis in the mammary gland of a cow (e.g., Palmquist, 2006). Medium-chain FA are those with 14 and 16 carbons and, although C14:0 mainly originates from de novo synthesis, C16:0 originates from 2 sources: approximately $50 \%$ from de novo synthesis and $50 \%$ from the diet of a cow. Most long-chain FA have 18 or more carbons and mainly originate from the cow's diet or from mobilization of body fat (e.g., Chilliard et al., 2000). In addition to differences in the number of carbons, FA may also differ in their degree of saturation. On average, more than $70 \%$ of the identified FA in milk consist are saturated FA and the remainder are unsaturated FA.

Variation in the content of several FA in milk is affected by genetic factors. Stoop et al. (2008) reported that individual milk FA have heritability estimates that range from 0.22 to 0.71 . Some well-characterized genes are recognized as having large effects on milk fat and FA composition, such as acyl-CoA:diacylglycerol acyltransferase1 (DGAT1), located on BTA14, and stearoyl-CoA desaturase1 (SCD1), located on BTA26 (e.g., Schennink et al., 2007, 2008). In addition, several regions of the bovine genome have been identified as having effects on milk fat and FA composition but have not yet been characterized (e.g., Bouwman et al., 2012). By fine-mapping these regions, it is possible to identify candidate genes (Ishii et al., 2013) associated with milk FA composition. Further insights into the biosynthesis of milk fat and FA are relevant if the aim is to change milk FA composition by means of breeding (Boichard and Brochard, 2012) or feeding strategies.

Fine-mapping allows refinement of genomic regions by testing a large number of SNP that are likely associated with a QTL (Hinds et al., 2005). Recently, a genomic region associated with short-chain FA in 
milk was detected on BTA17 (Bouwman et al., 2012). However, no candidate gene or causal variant has been identified so far. The aim of our study was to fine-map BTA17 with imputed 777,000 (777K) SNP genotypes to identify candidate genes associated with milk FA composition.

\section{MATERIALS AND METHODS}

This study is part of the Dutch Milk Genomics Initiative that aims to explore possibilities to modify milk FA composition through breeding. Bouwman et al. (2012) performed a genome-wide association study (GWAS) using 50,000 (50K) SNP genotypes based on milk FA composition of winter and summer milk samples. In the present study, we reanalyzed the same phenotypes and fine-mapped BTA17 using imputed $777 \mathrm{~K}$ SNP genotypes.

\section{Animals and Phenotypes}

Morning milk samples (500 mL/cow) were retrieved from 2,001 first-lactation Holstein-Friesian cows from 398 herds throughout the Netherlands. At least 3 cows per herd were sampled in 2 distinct seasons: February to March 2005 (winter samples) and May to June 2005 (summer samples). The milk samples were taken from the same cows during the same lactation. Some cows sampled in winter were no longer lactating when summer milk samples were taken. Additional cows were sampled from the same herds to guarantee milk samples from at least 3 cows per herd. A total of 1,905 cows had phenotypic records in winter, with each cow lactating between 63 and $282 \mathrm{~d}$ (see Stoop et al., 2008). A total of 1,795 cows had phenotypic records in the summer, with each cow lactating between 97 and $335 \mathrm{~d}$ (see Duchemin et al., 2013). About $50 \%$ of the cows in our experiment had access to pasture in summer (3.5 to $24 \mathrm{~h} / \mathrm{d}$ ), whereas all cows were kept indoors and fed silage in winter. Further details about the experimental design can be found in Stoop et al. (2008).

Milk FA composition was measured by GC at the COKZ laboratory (Qlip, Leudsen, the Netherlands). Milk fat was extracted from the milk samples, and FAME were prepared from fat fractions, as described by Schennink et al. (2007). The FA were identified and quantified by comparing the methyl ester chromatograms of the milk fat samples with the chromatograms of pure FAME standards (Stoop et al., 2008). The FA included in this study were measured as weight proportion of total fat (\% wt/wt) and are described in Table 1. In addition, an indicator of de novo synthesized milk FA was created by combining C6:0 through C14:0 individual FA in the index designated "C6:0-C14:0."

\section{Genotypes and Imputation}

A blood sample from each cow and semen from each bull were used to extract DNA. The DNA of 55 sires and 1,813 daughters belonging to our experimental population was genotyped with a $50 \mathrm{~K}$ SNP chip. This chip was designed by CRV (Arnhem, Netherlands), and was used to genotype the animals using the Infinium assay (Illumina Inc., San Diego, CA).

A reference population of 1,333 animals belonging to CRV and including the 55 sires with offspring in our data set was additionally genotyped with a $777 \mathrm{~K}$ SNP chip (Illumina Inc., San Diego, CA). This information on the reference population was used to impute the genotypes of our experimental population from $50 \mathrm{~K}$ to $777 \mathrm{~K}$ SNP. This imputation was done using Beagle version 3.2.2 (Browning and Browning, 2009) and resulted in a total of 1,736 animals being imputed to $777 \mathrm{~K}$ SNP. Of these 1,736 animals, 12 animals were excluded because of pedigree inconsistencies and 3 animals were subsequently excluded because their herds no longer met the requirement of a minimum of 3 animals sampled per herd. Consequently, 1,721 animals with imputed 777K SNP genotypes were available for this study. Imputation of BTA17 increased the number of SNP genotypes from 1,562 (i.e., 50K) to 22,240 (i.e., $777 \mathrm{~K})$. The positions of the imputed SNP were based on the bovine genome assembly UMD 3.1 (Zimin et al., 2009).

\section{Fine-Mapping of BTA17}

The fine-mapping of BTA17 was performed separately for winter and summer milk samples by using imputed 777K SNP genotypes and the 14 FA described in Table 1. For each season, animals were included in the analyses if both phenotypic and genotypic data were available. Therefore, 1,640 animals were available for winter milk, and 1,581 animals were available for summer milk samples.

Single SNP analyses were performed using the following animal model:

$$
\begin{aligned}
y_{i j k l m n o}= & \mu+b_{1} \times \operatorname{dim}_{i}+b_{2} \times e^{-0.05 \times \operatorname{dim}_{i}}+b_{3} \times a f c_{j} \\
& +b_{4} \times a f c_{j}^{2}+\text { season }_{k}+\text { scode }_{l} \\
& + \text { SNP }_{m}+\text { herd }_{n}+a_{o}+e_{i j k l m n o}
\end{aligned}
$$

where $y_{i j k l m n o}$ is the dependent variable; $\mu$ is the overall mean; $b_{1}$ and $b_{2}$ are the regression coefficients related to $\operatorname{dim}_{i} ; \operatorname{dim}_{i}$ is the covariate describing the effect of days in milk, modeled with a Wilmink curve (Wilmink, $1987) ; b_{3}$ and $b_{4}$ are the regression coefficients related to af $c_{j}$; $a f c_{j}$ is the covariate describing the effect of age at 
first calving; season $n_{k}$ is the fixed effect of calving season (June-August 2004, September-November 2004, or December 2004-February 2005); scode $e_{l}$ is the fixed effect accounting for differences in genetic level between groups of proven bull daughters and young bull daughters; $S N P_{m}$ is the fixed effect of SNP genotype; herd $d_{n}$ is the random effect of herd, and is assumed to be distributed as $N \sim\left(0, \mathbf{I} \sigma_{\text {herd }}^{2}\right)$, for which $\mathbf{I}$ is the identity matrix, and $\sigma_{\text {herd }}^{2}$ is the herd variance; $a_{o}$ is the random additive genetic effect of animal, and is assumed to be distributed as $N \sim\left(0, \mathbf{A} \sigma_{a}^{2}\right)$, where $\mathbf{A}$ is the additive genetic relationships matrix which consisted of 12,548 animals, and $\sigma_{a}^{2}$ is the additive genetic variance; and $e_{i j k l m n o}$ is the random residual effect, and is assumed to be distributed as $N \sim\left(0, \mathbf{I} \sigma_{e}^{2}\right)$, for which $\mathbf{I}$ is the identity matrix, and $\sigma_{e}^{2}$ is the residual variance.

Additive genetic and herd variances were estimated without the inclusion of SNP information, and the resulting estimates were fixed within model [1].

Heritability estimates were calculated from univariate analyses based on model [1] without the inclusion of $\mathrm{SNP}$ effects as follows: $h^{2}=\frac{\sigma_{a}^{2}}{\sigma_{a}^{2}+\sigma_{e}^{2}}$. Analyses were performed separately for winter and summer milk samples. All statistical analyses were performed using ASReml 3.0 (Gilmour et al., 2009).

\section{Construction of Haplotypes}

Haplotypes were constructed to further characterize a genomic region on BTA17, and these were constructed separately for winter and summer milk samples. This construction started with the identification of promising SNP by single-SNP analyses using model [1]. The SNP with the highest significance was defined as QTagSNP1. Subsequently, we corrected for the effect of QTagSNP1 by including QTagSNP1 as a fixed effect in model [1]. This correction allowed us to run a second round of single-SNP analyses and retrieve remaining significant SNP. After this second round of analyses, if another SNP still was significant, it was defined as QTagSNP2. In these analyses, a SNP was considered to be significant if $-\log _{10}(P$-value $) \geq 3$. Next, we corrected for the effects of QTagSNP2 in the model already extended with QTagSNP1 by further including QTagSNP2 as a fixed effect. This methodology was repeated until no additional significant SNP were retrieved. Linkage disequilibrium (LD) was estimated as the squared correlation $\left(\mathrm{r}^{2}\right)$ between all the identified QTagSNP using PLINK version 1.07 (Purcell et al., 2007). After the identification of QTagSNP, haplotypes were constructed based on the identified QTagSNP.
Effects of haplotypes were estimated with the following animal model:

$$
\begin{aligned}
y_{i j k l n p q r}= & \mu+b_{1} \times \operatorname{dim}_{i}+b_{2} \times e^{-0.05 \times \operatorname{dim}_{i}}+b_{3} \times a f c_{j} \\
& +b_{4} \times a f c_{j}^{2}+\text { season }_{k}+\text { scode }_{l} \\
& + \text { haplo1 }_{p}+\text { haplo }_{q}+\text { herd }_{n}+a_{r}^{*}+e_{i j k l n p q r},
\end{aligned}
$$

where variables are as previously described for model [1]; haplo $1_{p}$ is the random effect of the first haplotype; haplo $2_{q}$ is the random effect of the second haplotype, and they are both assumed to be distributed as $N \sim\left(0, \mathbf{I} \sigma_{\text {haplo }}^{2}\right)$, for which $\mathbf{I}$ is the identity matrix and $\sigma_{\text {haplo }}^{2}$ is the haplotype variance. The first and second haplotypes were jointly used to estimate one haplotype variance $\left(\sigma_{\text {haplo }}^{2}\right)$ and one effect for each haplotype. This was achieved by combining the design matrices of both haplotypes in ASReml; $\sigma_{r}^{*}$ is the random additive genetic effect of animal estimated without the inclusion of haplotypes and is assumed to be distributed as $\sim N\left(0, \mathbf{A} \sigma_{a^{*}}^{2}\right)$, for which $\mathbf{A}$ is the additive genetic relationships matrix, which consisted of 12,548 animals, and $\sigma_{a^{*}}^{2}$ is the additive genetic variance that remains after accounting for haplotype effects. The total additive genetic variance was defined as $\sigma_{a}^{2}=\sigma_{a^{*}}^{2}+\sigma_{\text {haplo* }}^{2}$. The fraction of genetic variance explained by haplotypes was defined as $\sigma_{\text {haplo }}^{2} / \sigma_{a}^{2}$.

Additionally, we tested whether predicted haplotype effects differed from each other. Significance levels of the differences between predicted effects of haplotypes were assessed using Student's $t$-tests, as implemented in ASReml. The predicted effect of a haplotype was considered significantly different from another haplotype if $P$-value $\leq 0.05$.

\section{RESULTS}

\section{Phenotypic Means and Heritability Estimates}

Phenotypic means and heritability estimates for milk FA composition in winter and summer milk samples are shown in Table 1. Winter milk had higher contents of short-chain FA than summer milk samples (14.2 vs. $13.7 \%$ ), higher contents of medium-chain FA (44.2 vs. $40.4 \%$ ), and lower contents of long-chain FA, such as C18:0 (8.7 vs. $9.9 \%$ ) and cis-9 C18:1 (18.2 vs. 20.5\%). Phenotypic variance was higher in summer compared with winter milk samples, but genetic variances were similar in both seasons. A detailed discussion on differences between winter and summer milk samples can be found in our previous study (Duchemin et al., 2013). 
Table 1. Phenotypic means (SD), and heritability estimates $\left(h^{2}\right)^{1}$ for individual FA based on 1,640 winter milk samples and 1,581 summer milk samples

\begin{tabular}{|c|c|c|c|c|}
\hline \multirow[b]{2}{*}{ Individual FA ( $\%$ wt $/ w t)$} & \multicolumn{2}{|c|}{ Winter } & \multicolumn{2}{|c|}{ Summer } \\
\hline & Mean (SD) & $h^{2}$ & Mean (SD) & $h^{2}$ \\
\hline \multicolumn{5}{|l|}{ Saturated FA } \\
\hline C4:0 & $3.51(0.27)$ & 0.47 & $3.52(0.35)$ & 0.41 \\
\hline $\mathrm{C} 6: 0$ & $2.23(0.16)$ & 0.46 & $2.17(0.21)$ & 0.39 \\
\hline C8:0 & $1.36(0.14)$ & 0.59 & $1.32(0.17)$ & 0.35 \\
\hline C10:0 & $3.02(0.43)$ & 0.73 & $2.87(0.45)$ & 0.48 \\
\hline C12:0 & $4.12(0.70)$ & 0.62 & $3.79(0.72)$ & 0.48 \\
\hline C14:0 & $11.62(0.92)$ & 0.62 & $11.16(1.05)$ & 0.54 \\
\hline C16:0 & $32.62(2.84)$ & 0.47 & $29.20(3.49)$ & 0.40 \\
\hline C18:0 & $8.71(1.39)$ & 0.28 & $9.86(1.77)$ & 0.19 \\
\hline \multicolumn{5}{|l|}{ Unsaturated FA } \\
\hline $\mathrm{C} 10: 1^{2}$ & $0.37(0.07)$ & 0.35 & $0.35(0.07)$ & 0.50 \\
\hline $\mathrm{C} 12: 1^{2}$ & $0.12(0.03)$ & 0.38 & $0.11(0.03)$ & 0.47 \\
\hline cis-9 C14: $1^{3}$ & $1.36(0.25)$ & 0.35 & $1.38(0.28)$ & 0.43 \\
\hline cis-9 C16:1 & $1.45(0.32)$ & 0.44 & $1.40(0.30)$ & 0.38 \\
\hline cis-9 C18: $1^{4}$ & $18.18(2.05)$ & 0.22 & $20.53(2.76)$ & 0.35 \\
\hline cis-9,trans-11 C18:2 (CLA) & $0.39(0.11)$ & 0.55 & $0.56(0.27)$ & 0.27 \\
\hline \multicolumn{5}{|c|}{$\begin{array}{l}{ }^{1} h^{2}=\sigma_{a}^{2} /\left(\sigma_{a}^{2}+\sigma_{e}^{2}\right) \text {, where } h^{2} \text { is the heritability estimate, } \sigma_{a}^{2} \text { is the additive genetic variance, and } \sigma_{e}^{2} \text { is the re- } \\
\text { sidual variance; SE between } 0.01 \text { and } 0.12 \text { for winter samples, and between } 0.02 \text { and } 0.08 \text { for summer samples. } \\
{ }^{2} \text { For } \mathrm{C} 10: 1 \text { and } \mathrm{C} 12: 1 \text {, the cis double bond could not be ascertained at the carbon } 9 \text { position. } \\
{ }^{3} \text { cis-9 } \mathrm{C} 14: 1 \text { represents the sum of } \text { cis-9 } \mathrm{C} 14: 1 \text { and iso } \mathrm{C} 15 \text { due to co-elution associated with the GC extraction } \\
\text { method. } \\
{ }^{4} \text { cis-9 } \mathrm{C} 18: 1 \text { represents the sum of } \text { cis-9 } \mathrm{C} 18: 1 \text { and trans-12 C18:1 due to co-elution associated with the GC } \\
\text { extraction method. }\end{array}$} \\
\hline
\end{tabular}

\section{Fine-Mapping of BTA17}

Results of the fine-mapping of BTA17 for winter and summer milk samples are shown in Supplemental Figure S1 (http://dx.doi.org/10.3168/jds.2014-8178). For both seasons, we analyzed associations between 22,240 imputed SNP and each of the 14 FA. In a region between 29.0 and $34.0 \mathrm{Mbp}$, multiple SNP showed highly significant associations with C6:0, C8:0, and C10:0. Moreover, multiple SNP showed associations both in winter and summer milk samples (Supplemental Figure S1). Previously, Bouwman et al. (2012) identified associations of multiple regions on BTA17 with C6:0, C8:0, C10:0, C14:1, and C16:1. Detailed analyses in the current study focused on the region between 29.0 and 34.0 Mbp because the strongest and most consistent associations were found in this region across winter and summer milk samples.

Figure 1A illustrates the strongest associations found with the imputed $777 \mathrm{~K}$ SNP genotypes for C8:0 in summer milk samples. Additionally in Figure 1A, these associations were overlaid with the associations found by Bouwman et al. (2012) using 50K SNP genotypes, which were mainly the same data as used in the current study. Within the marked region (Figure 1A), 10 significant SNP were found with the 50K SNP, whereas 83 significant SNP were found with the imputed $777 \mathrm{~K}$ SNP. The most significant SNP identified based on the imputed $777 \mathrm{~K}$ SNP $\left[-\log _{10}(P\right.$-value $\left.)=7.93\right]$ was not present on the 50K SNP array. The most significant SNP identified based on the 50K SNP genotypes was less significant $\left[-\log _{10}(P\right.$-value $)=6.21$; Bouwman et al., 2012] than the most significant SNP identified in the present study. The location of the QTL could be refined to the genomic region located between 29.0 and 34.0 Mbp on BTA17 (Figure 1A). Figure 1B shows the results of the associations for $5 \mathrm{FA}$ in summer milk samples for this region.

\section{Construction of Haplotypes}

The construction of haplotypes was based on the identified QTagSNP in the fine-mapping of BTA17. These SNP, QTagSNP1 and QTagSNP2, were different for winter and summer milk samples. For winter milk samples, QTagSNP1 was BovineHD1700008470 (rs109426433) located at $29.92 \mathrm{Mbp}$, and with minor allele frequency (MAF) of 0.47. QTagSNP1 was associated with $\mathrm{C} 6: 0\left[-\log _{10}(P\right.$-value $\left.)=4.90\right], \mathrm{C} 8: 0[-$ $\log _{10}(P$-value $\left.)=6.28\right], \mathrm{C} 10: 0\left[-\log _{10}(P\right.$-value $\left.)=4.03\right]$, and $\mathrm{C} 12: 0\left[-\log _{10}(P\right.$-value $\left.)=1.33\right]$. QTagSNP2 was BovineHD1700009150 (rs135934524) located at 32.90 Mbp, with MAF of 0.44. QTagSNP2 was associated with C6:0 $\left[-\log _{10}(P\right.$-value $\left.)=2.76\right), \mathrm{C} 8: 0\left[-\log _{10}(P-\right.$ value $)=3.27]$, and C10:0 $\left[-\log _{10}(P\right.$-value $\left.)=2.24\right]$. QTagSNP1 and QTagSNP2 showed the strongest associations with C8:0, and LD between QTagSNP1 and QTagSNP2 was $r^{2}=0.04$. 

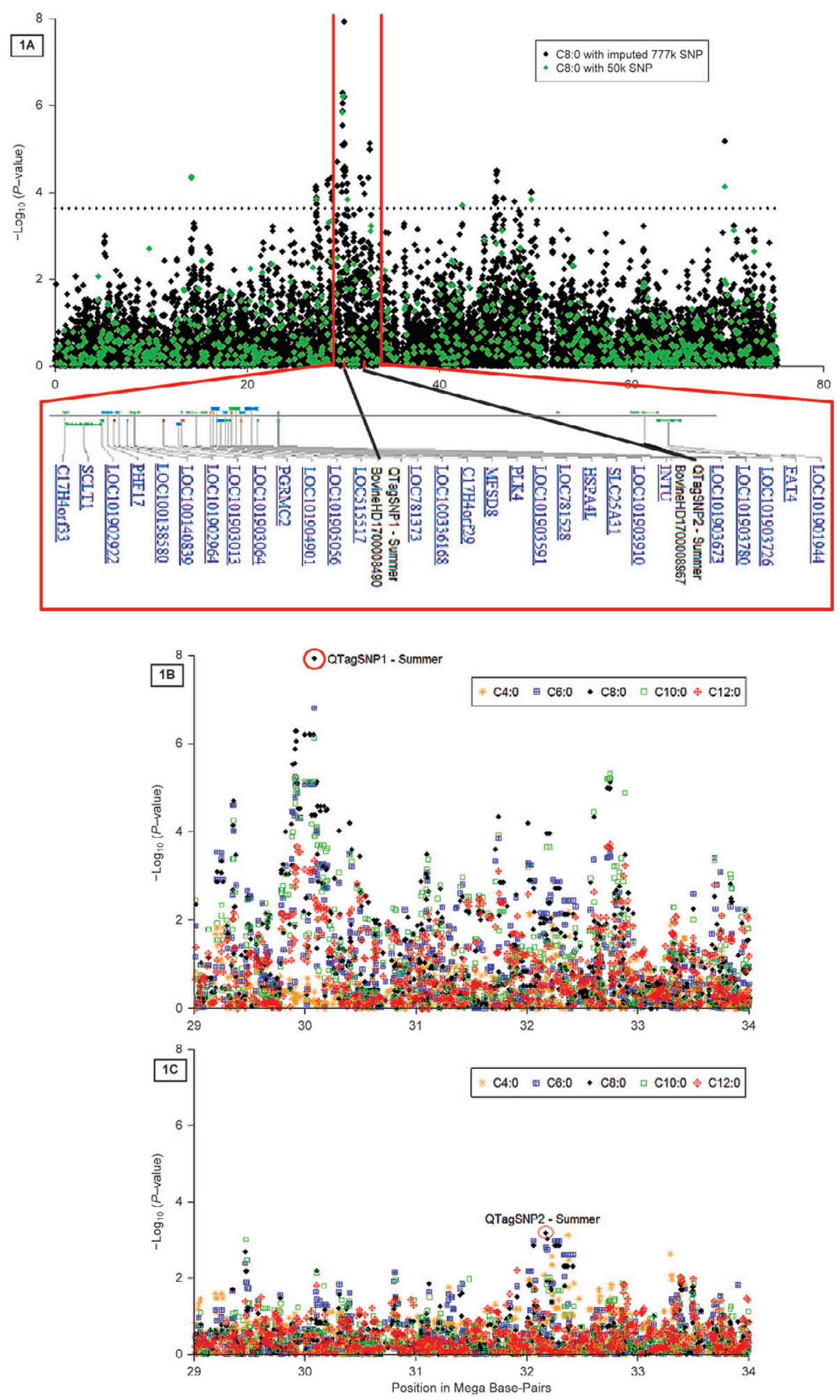

Figure 1. (A) Fine-mapping of BTA17 for C8:0 in summer milk samples showing genome-wide association of imputed 777K (777,000) SNP overlaid with genome-wide association of 50K (50,000) SNP genotypes done by Bouwman et al. (2012). The black dotted line is the genome-wide significance level based on 50K SNP genotypes at a false discovery rate of $0.05\left[-\log _{10}(P\right.$-value $\left.)=3.63\right]$. A list of candidate genes was added as well as an indication of the location of the SNP, with the highest significance referred to as QTagSNP1 and the SNP with the second highest significance referred to as QTagSNP2. (B) Fine-mapping of candidate region from 29.0 to 34.0 Mbp associated with C4:0 to C12:0 on BTA17 (results represent summer samples only). Circle indicates QTagSNP1. (C) Fine-mapping of candidate region on BTA17 after the correction for QTagSNP1 (results represent summer samples only). Circle indicates QTagSNP2. Color version available in the online PDF. 
For summer milk samples, QTagSNP1 was BovineHD1700008490 (rs109290136) located at 30.08 Mbp (Figure 1B), with MAF of 0.44. QTagSNP1 was associated with $\mathrm{C} 6: 0\left[-\log _{10}(P\right.$-value $\left.)=6.82\right], \mathrm{C} 8: 0[-$ $\log _{10}(P$-value $\left.)=7.93\right], \mathrm{C} 10: 0\left[-\log _{10}(P\right.$-value $\left.)=6.13\right]$, and $\mathrm{C} 12: 0\left[-\log _{10}(P\right.$-value $\left.)=3.35\right]$. QTagSNP2 was BovineHD1700008967 (rs135465158) located at 32.17 Mbp (Figure 1C), with MAF of 0.14. QTagSNP2 was associated with $\mathrm{C} 6: 0\left[-\log _{10}(P\right.$-value $\left.)=2.82\right], \mathrm{C} 8: 0[-$ $\log _{10}(P$-value $\left.)=3.19\right]$, and $\mathrm{C} 10: 0\left[-\log _{10}(P\right.$-value $)=$ 1.84]. QTagSNP1 and QTagSNP2 showed the strongest associations with C8:0. The LD between QTagSNP1 and QTagSNP2 was $r^{2}=0.07$. The LD between QTagSNP1 and all other markers in the fine-mapped region as well as significance of association with $\mathrm{C} 8: 0$ is represented in Supplemental Figure S2A (http://dx.doi.org/10.3168/ jds.2014-8178), and LD between QTagSNP2 and all other markers in the fine-mapped region as well as significance of association with $\mathrm{C} 8: 0$ is represented in Supplemental Figure S2B (http://dx.doi.org/10.3168/ jds.2014-8178).

Linkage disequilibrium between QTagSNP1 for winter milk samples and QTagSNP1 for summer milk samples was $\mathrm{r}^{2}=0.56$; LD among other combinations of QTagSNP based on winter or summer milk samples was low $\left(r^{2}<0.10\right)$.

For both winter and summer milk samples, 2 QTagSNP were identified. These 2 QTagSNP were used for haplotype construction, resulting in 4 haplotypes. As QTagSNP were not the same in winter and summer milk samples, different haplotypes were constructed for each season.

\section{Predicted Effects of Haplotypes}

Predicted effects of haplotypes are shown in Table 2. For winter samples, frequencies of haplotypes were 0.33 for A-A, 0.21 for A-G, 0.12 for C-A, and 0.35 for $\mathrm{C}-\mathrm{G}$. Whereas A-A haplotypes were associated with higher contents of C6:0, C8:0, C10:0, C12:0, C14:0, and the index C6:0-C14:0, C-G haplotypes were associated with lower contents of these FA and index. The absolute difference between one copy of the most contrasting haplotypes (A-A and C-G) was 0.040 for C6:0, 0.039 for C8:0, 0.090 for C10:0, 0.054 for C12:0, 0.065 for C14:0, and 0.239 for the index C6:0-C14:0. The fraction of genetic variance explained by haplotypes was $2.7 \%$ for C6:0, 2.8\% for C8:0, 1.4\% for C10:0, 0.5\% for C12:0, 0.3\% for C14:0, and 0.7\% for the index C6:0-C14:0.

In winter samples, predicted effects of the A-A haplotype differed significantly $(P \leq 0.05$; Table 2$)$ from effects of A-G, C-G, and C-A haplotypes for C6:0, C8:0, and C10:0. Effects of the C-G haplotype differed significantly $(P \leq 0.05)$ from effects of the A-G haplotype for C6:0, C8:0, and C10:0. Effects of the C-A haplotype did not differ from effects of the A-G haplotype for C6:0,

Table 2. Predicted effects of haplotypes (frequency given in parentheses after each haplotype) for de novo synthesized milk FA based on 1,640 winter milk samples and 1,581 summer milk samples

\begin{tabular}{|c|c|c|c|c|c|}
\hline \multirow[b]{2}{*}{ Trait } & \multicolumn{4}{|c|}{ Winter milk samples } & \multirow{2}{*}{$\begin{array}{c}\sigma_{\text {haplo }}^{2} / \sigma_{a}^{2} \\
(\%)^{1}\end{array}$} \\
\hline & A-A $(0.33)$ & A-G $(0.21)$ & C-A (0.12) & C-G (0.35) & \\
\hline $\mathrm{C} 4: 0$ & $0.000 \pm 0.000^{\mathrm{a}}$ & $0.000 \pm 0.000^{\mathrm{a}}$ & $0.000 \pm 0.000^{\mathrm{a}}$ & $0.000 \pm 0.000^{\mathrm{a}}$ & 0.0 \\
\hline C6:0 & $0.021 \pm 0.010^{\mathrm{a}}$ & $0.002 \pm 0.010^{\mathrm{b}}$ & $-0.004 \pm 0.011^{\mathrm{bc}}$ & $-0.019 \pm 0.010^{\mathrm{c}}$ & 2.7 \\
\hline $\mathrm{C} 8: 0$ & $0.020 \pm 0.009^{\mathrm{a}}$ & $0.002 \pm 0.009^{\mathrm{b}}$ & $-0.002 \pm 0.010^{\mathrm{b}}$ & $-0.019 \pm 0.009^{\mathrm{c}}$ & 2.8 \\
\hline C10:0 & $0.045 \pm 0.023^{\mathrm{a}}$ & $0.005 \pm 0.023^{\mathrm{b}}$ & $-0.005 \pm 0.025^{\mathrm{bc}}$ & $-0.045 \pm 0.023^{\mathrm{c}}$ & 1.4 \\
\hline $\mathrm{C} 12: 0$ & $0.026 \pm 0.020^{\mathrm{a}}$ & $0.004 \pm 0.020^{\mathrm{ab}}$ & $0.001 \pm 0.022^{\mathrm{ab}}$ & $-0.028 \pm 0.020^{\mathrm{b}}$ & 0.5 \\
\hline C14:0 & $0.037 \pm 0.028^{\mathrm{a}}$ & $0.003 \pm 0.028^{\mathrm{ab}}$ & $-0.012 \pm 0.031^{\mathrm{ab}}$ & $-0.028 \pm 0.028^{\mathrm{b}}$ & 0.3 \\
\hline C6:0-C14:0 & $0.106 \pm 0.084^{\mathrm{a}}$ & $0.027 \pm 0.085^{\mathrm{ab}}$ & $0.000 \pm 0.091^{\mathrm{ab}}$ & $-0.133 \pm 0.084^{\mathrm{b}}$ & 0.7 \\
\hline
\end{tabular}

Summer milk samples

\begin{tabular}{|c|c|c|c|c|c|}
\hline & A-G $(0.44)$ & A-A (012) & C-A (0.01) & C-G $(0.42)$ & \\
\hline $\mathrm{C} 4: 0$ & $-0.009 \pm 0.009^{\mathrm{a}}$ & $0.002 \pm 0.010^{\mathrm{a}}$ & $0.006 \pm 0.011^{\mathrm{a}}$ & $0.000 \pm 0.009^{\mathrm{a}}$ & 0.3 \\
\hline C6:0 & $-0.043 \pm 0.021^{\mathrm{a}}$ & $-0.009 \pm 0.022^{\mathrm{b}}$ & $0.046 \pm 0.027^{\mathrm{c}}$ & $0.005 \pm 0.021^{\mathrm{bc}}$ & 9.7 \\
\hline $\mathrm{C} 8: 0$ & $-0.035 \pm 0.016^{\mathrm{a}}$ & $-0.003 \pm 0.017^{\mathrm{b}}$ & $0.030 \pm 0.021^{\mathrm{b}}$ & $0.008 \pm 0.016^{\mathrm{b}}$ & 9.0 \\
\hline C10:0 & $-0.068 \pm 0.031^{\mathrm{a}}$ & $0.003 \pm 0.033^{\mathrm{b}}$ & $0.030 \pm 0.043^{\mathrm{b}}$ & $0.034 \pm 0.032^{\mathrm{b}}$ & 3.5 \\
\hline C12:0 & $-0.049 \pm 0.032^{\mathrm{a}}$ & $0.003 \pm 0.035^{\mathrm{ab}}$ & $-0.006 \pm 0.045^{\mathrm{ab}}$ & $0.052 \pm 0.033^{\mathrm{b}}$ & 1.8 \\
\hline C14:0 & $-0.055 \pm 0.039^{\mathrm{a}}$ & $0.004 \pm 0.044^{\mathrm{ab}}$ & $0.000 \pm 0.054^{\mathrm{ab}}$ & $0.051 \pm 0.041^{\mathrm{b}}$ & 0.9 \\
\hline $\mathrm{C} 6: 0-\mathrm{C} 14: 0$ & $-0.329 \pm 0.168^{\mathrm{a}}$ & $-0.050 \pm 0.180^{\mathrm{b}}$ & $0.213 \pm 0.237^{\mathrm{b}}$ & $0.166 \pm 0.172^{\mathrm{b}}$ & 5.0 \\
\hline
\end{tabular}


C8:0, and C10:0, but did differ significantly $(P \leq 0.05)$ from effects of the C-G haplotype for C8:0. These results suggest that 2 groups of haplotypes with distinct effects (A-A and A-G/C-A/C-G) exist for C6:0 and $\mathrm{C} 10: 0$, and that 3 groups of haplotypes with distinct effects (A-A, C-G, and A-G/C-A) exist for C8:0.

For summer samples, frequencies of haplotypes were 0.44 for A-G, 0.12 for A-A, 0.01 for C-A, and 0.42 for C-G. Whereas C-G haplotypes were associated with higher contents of C6:0, C8:0, C10:0, C12:0, C14:0, and the index $\mathrm{C} 6: 0-\mathrm{C} 14: 0, \mathrm{~A}-\mathrm{G}$ haplotypes were associated with lower contents of these FA and index. The absolute difference between one copy of the most contrasting haplotypes (C-G and A-G) was 0.048 for C6:0, 0.043 for C8:0, 0.102 for C10:0, 0.101 for C12:0, 0.106 for $\mathrm{C} 14: 0$, and 0.495 for the index C6:0-C14:0. The fraction of genetic variance explained by haplotypes was $0.3 \%$ for $\mathrm{C} 4: 0,9.7 \%$ for $\mathrm{C} 6: 0,9 \%$ for $\mathrm{C} 8: 0$, $3.5 \%$ for $\mathrm{C} 10: 0,1.8 \%$ for $\mathrm{C} 12: 0,0.9 \%$ for $\mathrm{C} 14: 0$, and $5.0 \%$ for the index C6:0-C14:0.

In summer samples, predicted effects of the A-G haplotype differed significantly $(P \leq 0.05$; Table 2$)$ from effects of A-A, C-G, and C-A haplotypes for C6:0, C8:0, C10:0, and the index C6:0-C14:0. Additionally, effects of the A-G haplotype differed significantly $(P \leq 0.05)$ from effects of the C-G haplotype for C12:0 and C14:0. Effects of the C-G haplotype did not differ from the effects of C-A and A-A haplotypes for any of the traits. These results suggest that 2 groups of haplotypes have distinct effects (A-G and A-A/C-A/C-G) for C6:0, C8:0, C10:0, C12:0, C14:0, and the index C6:0-C14:0.

\section{DISCUSSION}

In the present study, we refined the location of a QTL first described by Bouwman et al. (2012). This QTL seems to influence multiple de novo synthesized FA. We fine-mapped BTA17 by using imputed $777 \mathrm{~K}$ SNP genotypes and by using winter and summer milk FA composition. To further characterize the effects associated with this genomic region, we constructed haplotypes for each season.

\section{Fine-Mapping of BTA17}

The fine-mapping of BTA17 combined high-density SNP genotyping with imputation. Imputation was based on a large reference population genotyped with $777 \mathrm{~K}$ SNP. Additionally, the 55 sires belonging to our experimental population were genotyped with both $50 \mathrm{~K}$ and $777 \mathrm{~K}$ SNP arrays. Our experimental population, which was composed of the daughters of the 55 sires, was imputed from $50 \mathrm{~K}$ to $777 \mathrm{~K}$ SNP genotypes using Beagle software (Browning and Browning, 2009). The estimated error of this imputation was $<1 \%$. Pausch et al. (2013) showed that imputation to high-density genotypes largely depends on the size of the reference population. An imputation accuracy of about $99 \%$ can be obtained when a reference population of more than 400 animals is used (Pausch et al., 2013). This is in line with the imputation accuracy obtained in the current study. When imputation accuracy is high, GWAS based on imputed genotypes can assist in fine-mapping because imputation provides a high-resolution view of an associated region and increases the chance that a causal SNP can be directly identified (Marchini and Howie, 2010). In the present study, the number of SNP increased by at least 10 times with the imputation of BTA17 from 50K to 777K SNP genotypes.

The GWAS by Bouwman et al. (2012) with 50K SNP genotypes identified a QTL associated with milk FA composition on BTA17. By fine-mapping BTA17 with the imputed $777 \mathrm{~K}$ SNP genotypes, additional SNP were found to be significantly associated with milk FA, and these were more significant than the SNP found by Bouwman et al. (2012). In addition, multiple FA showed associations with the same genomic region on BTA17, in both winter and summer milk samples (Supplemental Figure S1; http://dx.doi.org/10.3168/ jds.2014-8178). We focused on the strongest and most consistent associations found in winter and summer milk samples. These associations were identified in a region located between 29 and $34 \mathrm{Mbp}$. Additional analyses in which we extended the region (26-34 Mbp) showed results comparable to those presented herein.

Within this genomic region, summer milk showed more pronounced associations than winter milk samples. Duchemin et al. (2013) reported strong genetic correlations between winter and summer milk fat composition of de novo synthesized FA (e.g., 0.95 for C6:0, 0.93 for $\mathrm{C} 8: 0$, and 0.95 for C10:0). These strong genetic correlations suggest that de novo FA in winter and in summer milks are genetically the same trait. In addition, GWAS by Bouwman et al. (2012) showed that many genomic regions associated with milk FA in winter milk could be confirmed in summer milk samples (e.g., BTA17). Therefore, it is likely that milk FA composition is influenced by similar groups of genes. When studying the effects of DGAT1 polymorphism on milk fat composition in winter and summer milk samples, Duchemin et al. (2013) concluded that the genotypic effects were in the same direction, but that some of the genotypic effects were larger in summer compared with winter. Duchemin et al. (2013) concluded that differences between winter and summer milk fat composition were likely due to differences in the diets of the cows and that the effects of DGAT1 were scaled. This scaling resulted in a significant DGAT1 by season interaction, 
especially for short-chain FA (C4:0 to C14:0). In the present study, similar scaling effects might explain the more pronounced associations found in summer compared with winter milk samples.

\section{Construction of Haplotypes}

Haplotypes were constructed by first retrieving the most significant SNP within the fine-mapped region. This SNP, QTagSNP1, was associated with C8:0. Most of the variation in the region was explained by QTagSNP1, but not all. The remaining variation was accounted for by QTagSNP2 (results shown for summer samples, Figure 1B and 1C). After adjusting for both QTagSNP, no other significant SNP was found. Based on the 2 QTagSNP, 4 haplotypes were constructed. In summer milk samples, these haplotypes explained almost $10 \%$ of the genetic variance in $\mathrm{C} 6: 0,9 \%$ in $\mathrm{C} 8: 0$, $3.5 \%$ in $\mathrm{C} 10: 0,1.8 \%$ in $\mathrm{C} 12: 0$, and $0.9 \%$ in $\mathrm{C} 14: 0$ (Table $2)$. When these FA were combined into an index, haplotypes explained $5 \%$ of the genetic variance in de novo synthesized milk FA (C6:0-C14:0; Table 2).After testing for differences between these haplotypes, we concluded that estimated effects in summer milk for 3 out of 4 haplotypes did not differ from each other. Therefore, our 4 haplotypes could be divided in 2 groups with distinct effects on C6:0, C8:0, C10:0, C12:0, C14:0, and the index $\mathrm{C} 6$ :0-C14:0: A-G versus the remaining haplotypes. The existence of 2 groups of haplotypes with distinct effects could be explained by 1 causal variant; that is, 1 QTL. However, we cannot exclude the presence of multiple causal variants in strong LD.

The QTL region is associated with multiple de novo synthesized FA. De novo synthesis occurs within the mammary gland of a cow and is a process that elongates precursors by adding C2:0. These precursors originate from blood lipids and can be acetate $(\mathrm{C} 2: 0)$, propionate (C3:0), or butyrate (C4:0). Butyrate in milk may originate from de novo synthesis or directly from $\beta$-hydroxybutyrate derived from the blood (e.g., Craninx et al., 2008). Depending on the precursor, the elongation process ends at either $\mathrm{C} 16: 0$ or C17:0. Results of the current study show that predicted effects of haplotypes increase from C6:0 to C14:0; however, the proportion of genetic variance explained by haplotypes decreases from C6:0 to C14:0. This increase of haplotype effects tends to be more pronounced in summer than in winter milk samples (Table 2). These results suggest that our candidate gene is involved in the elongation of FA or the early termination of this process (Barber et al., 1997), and it might be upregulated in summer compared with winter milk samples.

Interestingly, in other species, such as humans, macaques, and pigs, this genomic region is highly con- served. Further, in dairy cattle breeds, 2 studies suggested that this region on BTA17 contains signatures of selection: Qanbari et al. (2011) identified signatures of selection in a region close to the PGRMC2 gene (progesterone receptor membrane component 2) at 29.8 Mbp; and Stella et al. (2010) in a region close to the SPRY1 gene [sprout homolog1, antagonist of FGF signaling (Drosophila)] at $34.7 \mathrm{Mbp}$. This genomic region might be related to a highly conserved evolutionary mechanism.

\section{Candidate Genes}

Information on candidate genes possibly associated with de novo synthesized FA was retrieved from the National Center for Biotechnology Information website (http://www.ncbi.nlm.nih.gov/). The QTL region on BTA17 contains 29 genes, but 18 of these have not yet been characterized. Between QTagSNP1 and QTagSNP2 in summer samples are 11 genes, of which 5 have been characterized (Figure 1A).

The characterized gene that is closest to the most significant association is PGRMC2, which is located between 29.87 and $29.89 \mathrm{Mbp}$. This gene belongs to the superfamily cytochrome b5-like heme/steroid binding domain. This superfamily is involved in the FA metabolic process and oxido-reductase activity. In humans, this gene has been associated with breast adenocarcinoma (Causey et al., 2011), and it was identified as a regulator of cytochrome $\mathrm{P} 450$ enzyme activity (Wendler and Wehling, 2013). By sequencing the mRNA found in the milk fat layer, Lemay et al. (2013) showed that PGRMC2 is expressed in humans throughout lactation, including in colostrum, transitional, and mature milk.

In cattle, PGRMC2 has been associated with fertility. Kowalik et al. (2013) showed that expression of PGRMC2 mRNA in the bovine endometrium was higher in the first trimester of pregnant cows compared with cyclic animals. However, the translation of PGRMC2 mRNA into protein within the bovine endometrium was not different between cyclic and pregnant cows. In our study, cows in the winter and summer sampling periods were in different stages of lactation (average of $166 \mathrm{~d}$ in winter and average of $247 \mathrm{~d}$ in summer samples) and probably at different stages of pregnancy. This might explain the more pronounced associations found in summer milk samples. Therefore, we performed additional analyses in which we investigated interactions between stage of lactation and our QTagSNP in both seasons. None of these interactions was significant (results not shown). Bionaz et al. (2012) showed that PGRMC2 is expressed during lactation in bovine mammary tissue, although PGRMC2 has not been associated with milk FA composition in dairy cattle. 
Of the genes located within our QTL region, Bionaz et al. (2012) showed that 4 other genes are highly expressed during lactation in bovine mammary tissue: UPF0462 protein C4orf33-like (LOC513251), sodium channel and clathrin linker 1 (SCLT1), la-related protein 1B-like (LOC515517), and chromosome 17 open reading frame, human C4orf29 ( $\mathrm{C}_{17} \mathrm{H}_{4}$ orf29). The location for these genes is between 29.10 and 29.12 Mbp for LOC513251, between 29.12 and 29.35 Mbp for SCLT1, between 30.03 and 30.07 Mbp for LOC515517, and between 30.10 and $30.13 \mathrm{Mbp}$ for C17H4orf29. By sequencing the mRNA found in milk fat layer, Lemay et al. (2013) showed in humans that 3 of these 4 genesC17h4orf33 (validated LOC513251 gene in humans), $L A R P 1 B$ (validated LOC515517 gene in humans), and C17H4orf29 - are expressed during all stages of lactation. These 4 genes have not yet been associated with milk FA composition.

In the present paper, we refined the location of a QTL associated with multiple de novo synthesized milk FA to a region between 29.0 and $34.0 \mathrm{Mbp}$ on BTA17. We characterized the effects associated with this region by constructing haplotypes and identified candidate genes possibly related to this QTL.

\section{CONCLUSIONS}

The fine-mapping of BTA17 improved the location of a QTL associated with multiple de novo synthesized milk FA. In summer milk samples, this QTL region explained a large proportion of the genetic variance in these FA individually (e.g., 10\% in C6:0). When all de novo synthesized milk FA were combined into an index, this QTL region explained $5 \%$ of the genetic variance. This QTL region seems to be involved in either the elongation process of the de novo FA synthesis or in the early termination of this process. In addition, the effects of this QTL region were greater in summer compared with winter milk samples. Candidate genes associated with milk FA composition could not be clearly identified for this QTL because the QTL region on BTA17 is still being characterized. A characterized gene that might be of interest within the QTL region is PGRMC2.

\section{ACKNOWLEDGMENTS}

This study is part of the Dutch Milk Genomics Initiative, funded by Wageningen University, the Dutch Dairy Association (NZO; Zoetermeer, the Netherlands), Cooperative Cattle Improvement Organization (CRV; Arnhem, the Netherlands), and the Dutch Technology Foundation (STW; Utrecht, the Netherlands). We thank Chris Schrooten from CRV (Arnhem, the Netherlands) for the imputation of the $777 \mathrm{~K}$ SNP genotypes. The first author currently benefits from a joint grant from the European Commission [within the framework of the Erasmus-Mundus joint doctorate "EGS-ABG" (Paris, France) and Breed4Food (a public-private partnership in the domain of animal breeding and genomics and CRV].

\section{REFERENCES}

Barber, M. C., R. A. Clegg, M. T. Travers, and R. G. Vernon. 1997. Lipid metabolism in the lactating mammary gland. Biochim. Biophys. Acta 1347:101-126.

Bionaz, M., K. Periasamy, S. L. Rodriguez-Zas, W. L. Hurley, and J. J. Loor. 2012. A novel dynamic impact approach DIA for functional analysis of time-course omics studies: validation using the bovine mammary transcriptome. PLoS ONE 7:e32455.

Boichard, D., and M. Brochard. 2012. New phenotypes for new breeding goals in dairy cattle. Animal 6:544-550.

Bouwman, A. C., M. H. P. W. Visker, J. A. M. van Arendonk, and H. Bovenhuis. 2012. Genomic regions associated with bovine milk fatty acids in both summer and winter milk samples. BMC Genet. 13:93.

Browning, B. L., and S. R. Browning. 2009. A unified approach to genotype imputation and haplotype-phase inference for large data sets of trios and unrelated individuals. Am. J. Hum. Genet. 84:210-223.

Causey, M. W., L. J. Huston, D. M. Harold, C. J. Charaba, D. L. Ippolito, Z. S. Hoffer, T. A. Brown, and J. D. Stallings. 2011. Transcriptional analysis of novel hormone receptors PGRMC1 and PGRMC2 as potential biomarkers of breast adenocarcinoma staging. J. Surg. Res. 171:615-622.

Chilliard, Y., A. Ferlay, R. M. Mansbridge, and M. Doreau. 2000 Ruminant milk fat plasticity: Nutritional control of saturated, polyunsaturated, trans and conjugated fatty acids. Ann. Zootech. 49:181-206.

Craninx, M., A. Steen, H. Van Laar, T. Van Nespen, J. Martin-Tereso, B. De Baets, and V. Fievez. 2008. Effect of lactation stage on the odd- and branched-chain milk fatty acids of dairy cattle under grazing and indoor conditions. J. Dairy Sci. 91:2662-2677.

Duchemin, S., H. Bovenhuis, W. M. Stoop, A. C. Bouwman, J. A. M. van Arendonk, and M. H. P. W. Visker. 2013. Genetic correlation between composition of bovine milk fat in winter and summer, and DGAT1 and SCD1 by season interactions. J. Dairy Sci. 96:592-604.

Gilmour, A. R., B. Gogel, B. Cullis, and R. Thompson. 2009. ASReml User Guide. Release 3.0. VSN International Ltd., Hemel Hempstead, UK.

Hinds, D. A., L. L. Stuve, G. B. Nilsen, E. Halperin, E. Eskin, D. G. Ballinger, K. A. Frazer, and D. R. Cox. 2005. Whole-genome patterns of common DNA variation in three human populations. Science 307:1072-1079.

Ishii, A., K. Yamaji, Y. Uemoto, N. Sasago, E. Kobayashi, N. Kobayashi, T. Matsuhashi, S. Maruyama, H. Matsumoto, S. Sasazaki, and H. Mannen. 2013. Genome-wide association study for fatty acid composition in Japanese Black cattle. Anim. Sci. J. 84:675-682.

Jensen, R. G. 2002. The composition of bovine milk lipids: January 1995 to December 2000. J. Dairy Sci. 85:295-350.

Kowalik, M. K., D. Slonina, R. Rekawiecki, and J. Kotwica. 2013. Expression of progesterone receptor membrane component (PGRMC) 1 and 2, serpine mRNA binding protein 1 (SERBP1) and nuclear progesterone receptor (PGR) in the bovine endometrium during the estrous cycle and the first trimester of pregnancy. Reprod. Biol. 13:15-23.

Lemay, D. G., O. A. Ballard, M. A. Hughes, A. L. Morrow, N. D. Horseman, and L. A. Nommsen-Rivers. 2013. RNA sequencing of the human milk fat layer transcriptome reveals distinct gene expression profiles at three stages of lactation. PLoS ONE 8:e67531. 
Marchini, J., and B. Howie. 2010. Genotype imputation for genomewide association studies. Nat. Rev. Genet. 11:499-511.

Palmquist, D. L. 2006. Milk fat: Origin of fatty acids and influence of nutritional factors thereon. Pages 43-92 in Advanced Dairy Chemistry: Lipids. Vol. 2. P. F. Fox and P. L. H. McSweeney, ed. Springer, New York, NY.

Pausch, H., B. Aigner, R. Emmerling, C. Edel, K.-U. Götz, and R. Fries. 2013. Imputation of high-density genotypes in the Fleckvieh cattle population. Genet. Sel. Evol. 45:3.

Purcell, S., B. Neale, K. Todd-Brown, L. Thomas, M. A. Ferreira, D. Bender, J. Maller, P. Sklar, P. I. De Bakker, and M. J. Daly. 2007. PLINK: A tool set for whole-genome association and populationbased linkage analyses. Am. J. Hum. Genet. 81:559-575.

Qanbari, S., D. Gianola, B. Hayes, F. Schenkel, S. Miller, S. Moore, G. Thaller, and H. Simianer. 2011. Application of site and haplotypefrequency based approaches for detecting selection signatures in cattle. BMC Genomics 12:318.

Schennink, A.. J. M. L. Heck, H. Bovenhuis, M. H. P. W. Visker, H. J. F. van Valenberg, and J. A. M. van Arendonk. 2008. Milk fatty acid unsaturation: Genetic parameters and effects of stearoyl-CoA desaturase $(S C D 1)$ and acyl CoA:diacylglycerol acyltransferase 1 (DGAT1). J. Dairy Sci. 91:2135-2143.
Schennink, A., W. Stoop, M. Visker, J. Heck, H. Bovenhuis, J. Van Der Poel, H. Van Valenberg, and J. Van Arendonk. 2007. DGAT1 underlies large genetic variation in milk-fat composition of dairy cows. Anim. Genet. 38:467-473.

Stella, A., P. Ajmone-Marsan, B. Lazzari, and P. Boettcher. 2010. Identification of selection signatures in cattle breeds selected for dairy production. Genetics 185:1451-1461.

Stoop, W. M., J. A. M. van Arendonk, J. M. L. Heck, H. J. F. van Valenberg, and H. Bovenhuis. 2008. Genetic parameters for major milk fatty acids and milk production traits of Dutch HolsteinFriesians. J. Dairy Sci. 91:385-394.

Wendler, A., and M. Wehling. 2013. PGRMC2, a yet uncharacterized protein with potential as tumor suppressor, migration inhibitor, and regulator of cytochrome P450 enzyme activity. Steroids $78: 555-558$.

Wilmink, J. B. M. 1987. Adjustment of test-day milk, fat and protein yield for age, season and stage of lactation. Livest. Prod. Sci. $16: 335-348$.

Zimin, A. V., A. L. Delcher, L. Florea, D. R. Kelley, M. C. Schatz, D. Puiu, F. Hanrahan, G. Pertea, C. P. Van Tassell, and T. S. Sonstegard. 2009. A whole-genome assembly of the domestic cow, Bos taurus. Genome Biol. 10:R42. 